\title{
Pretreatment Neutrophil-To-Lymphocyte Ratio (NLR) as a Prog- nostic Factor of Outcome for Patients with Oral Cavity Squamous Cell Carcinoma: A Single Institution Experience
}

\author{
Ashraf Farrag ${ }^{1,2^{*}}$, Arif Shaukat ${ }^{1}$, Muhammad Ali ${ }^{1}$ and Magdy Kandil ${ }^{1}$ \\ ${ }^{1}$ Department of Oncology, Prince Sultan Military Medical City, Riyadh, Kingdom of Saudi Arabia \\ ${ }^{2}$ Clinical Oncology Department, Assiut University, Assiut, Egypt
}

*Corresponding author: Ashraf Farrag, Department of Oncology, Prince Sultan Military Medical City, Riyadh, PO.Box 7897 Riyadh 11159, Kingdom of Saudi Arabia

\begin{abstract}
Background: Pretreatment Neutrophil-to-lymphocyte ratio (NLR) has been correlated with survival outcome in different types of cancers. In this study we evaluated the prognostic significance of pretreatment NLR in patients with oral squamous cell carcinoma (OSCC).

Methods: We retrospectively collected the clinical and epidemiological data of all biopsy proven, non-metastatic OSCC treated in our center between 2009-2015. Pretreatment absolute neutrophils and lymphocytes counts used to obtain NLR. The impact of NLR on overall (OS) and progression free survivals (PFS) was investigated in both uniand multivariate analyses.

Results: We identified a total of 68 patients of OSCC with a median pretreatment NLR ratio of 1.79. Patients were classified into two groups; high NLR and low NLR based on median NLR as a cut-off. Elevated NLR was significantly associated with male gender $(p=0.003)$ and with the presence lymph node metastases ( $p=0.029$ ). The 3 -years OS and PFS for low and high NLR groups were $(69.5 \%$ vs. $54.7 \%$, $p=0.037)$ and $(51 \%$ vs. $29.5 \%, p=0.028)$, respectively. In multivariate analysis NLR was significant for both OS ( $p=$ $0.005)$ and PFS ( $p=0.008)$.

Conclusions: Pretreatment NLR has been shown to be an independent prognostic factor for our patients with OSCC. This provides a simple and easily measurable indicator of outcome. Larger and prospective studies are required to determine the optimal cut-off values to accurately identify NLR related risk groups.
\end{abstract}

\section{Keywords}

Neutrophils-to-lymphocyte ratio, Oral cancer, Prognostic factor

\section{Introduction}

Oral cancer is the most common head and neck cancer (HNC). Its Global incidence in 2015 was 410,000 cases with 146,000 deaths [1]. Despite recent advances in treatment of oral squamous cell carcinoma (OSCC), treatment failure remains high with unsatisfactory 5-y survival rates [2].

Prognosis in OSCC depends on multiple variables including clinical, histological, and biochemical factors. Recognition of HPV status as a predictor and prognostic factor for improved outcomes recently has led to proposed changes in treatment algorithm for OSCC management. Other factors such as depth of invasion led to changes in the recent TNM staging. However, like other HNC, it is still challenging to predict treatment outcome even in patients with the same TNM stage [3]. This warrants the search for more prognostic as well as predictive factors in order to identify patients in need of intensified or innovative types of treatment.

Several oral inflammatory conditions such as submucous fibrosis, lichen planus, and chronic ulcers are implicated in the pathogenesis of OSCC. It was proven that persistent inflammation promotes cell proliferation and may lead to oncogenic activation and tumor suppressor genes inactivation. This will result in genomic insatiability and promotes malignant transformation. In addition, inflammatory factors mediate the development of a tumor-associated stoma. Activated cells in

Citation: Farrag A, Shaukat A, Ali M, Kandil M (2018) Pretreatment Neutrophil-To-Lymphocyte Ratio (NLR) as a Prognostic Factor of Outcome for Patients with Oral Cavity Squamous Cell Carcinoma: A Single Institution Experience. Int J Cancer Clin Res 5:100. doi.org/10.23937/2378-3419/1410100 Accepted: November 08, 2018; Published: November 10, 2018

Copyright: (C) 2018 Farrag A, et al. This is an open-access article distributed under the terms of the Creative Commons Attribution License, which permits unrestricted use, distribution, and reproduction in any medium, provided the original author and source are credited. 
this stoma mediate angiogenesis, immune evasion and metastasis [4].

Neutrophils-to-Lymphocytes ratio (NLR) as an expression of systemic inflammatory response to causative agents has shown correlation with outcomes in several malignancies. Breast cancer [5], gastric cancer [6], ovarian cancer [7], Renal cell carcinoma [8], lung cancer [9] and nasopharyngeal cancer [10] are few of the examples. NLR can provide an early and simple indicator for outcome compared with other prognostic factors that would be available only after surgical resection or require advanced laboratory techniques.

There are few studies exploring the prognostic effect of NLR in OSCC but the results have been conflicting. Although prognostic impact of NLR in OSCC was shown in some studies $[11,12]$ but similar correlation was not confirmed by others [13]. We conducted this review to explore the significance of the correlation between NLR and overall survival in our patients with SCC of oral cavity.

\section{Patients and Methods}

\section{Patients' selection}

A retrospective review of all patients' notes with pathologically proven oral cavity cancer treated radically, single or multimodality treatment, during 2009-2015 at our facility. Patients with non-squamous histology, distant metastases at presentation, patients with incomplete data or patients who started any oncological treatment outside our center were excluded from the study. Patients with clinical evidence of infection or other inflammatory conditions or with history of recent steroid intake were also excluded.
Finally we collected 68 non-metastatic squamous cell carcinoma of the oral cavity. Demographic data and clinical characteristics were collected for all cases from medical records. TNM staging was reported according to the 2010 AJCC system.

Information about pretreatment hematologic parameters was collected. NLR was calculated as the ratio of absolute neutrophil count to absolute lymphocyte count.

\section{Treatment and follow up}

All patients were subjected to standard treatment protocols according to primary tumor site, stage and patients general conditions. After receiving primary treatment, patients were followed in the combined oncology and head and neck surgery clinic. Follow up visits were every 3 months for the first year, every 4 months in the second year and every 6 months till completing 5 years, then annually. Clinical examination was performed at each visit. Radiological evaluation of response usually performed after the first post-treatment visit by computed tomography (CT), Positron Emission Tomography (PET-CT) or Magnetic Resonance Imaging (MRI) as per agreed protocol. Imaging studies were repeatedwhen necessary for restaging and to rule out locoregional recurrence. Local or regional recurrence had to have pathological confirmation prior to any other treatment as appropriate.

\section{Statistical Analysis}

Survival times were calculated from the day of diagnosis. Overall survival (OS) and Progression free survival (PFS) were calculated at the date of death or relapse or the last date of follow up. Kaplan-Meier method was

Table 1: Correlation between NLR and different variables. High NLR (> 1.79) and Low NLR $(<1.79)$.

\begin{tabular}{|c|c|c|c|c|c|}
\hline Variables & & & Low NLR & High NLR & $P$ value \\
\hline \multirow[t]{2}{*}{ AGE } & $<65$ & $41(60 \%)$ & 21 & 20 & 0.76 \\
\hline & $\geq 65$ & $27(40 \%)$ & 12 & 15 & \\
\hline \multirow[t]{2}{*}{ SEX } & M & $44(65 \%)$ & 15 & 29 & 0.003 \\
\hline & $\mathrm{F}$ & $24(35 \%)$ & 18 & 6 & \\
\hline \multirow[t]{2}{*}{ PS } & $<2$ & $43(63 \%)$ & 20 & 23 & 0.85 \\
\hline & $\geq 2$ & $25(37 \%)$ & 13 & 12 & \\
\hline \multirow[t]{3}{*}{ Tobacco } & Yes & $39(57 \%)$ & 19 & 20 & 0.86 \\
\hline & NO & $13(19 \%)$ & 7 & 6 & \\
\hline & N/A & $16(24 \%)$ & 7 & 9 & \\
\hline \multirow[t]{2}{*}{ Primary site } & Tongue & $35(51 \%)$ & 18 & 17 & 0.45 \\
\hline & Others & $33(49 \%)$ & 13 & 20 & \\
\hline \multirow[t]{2}{*}{ LN status } & Positive & $35(51 \%)$ & 12 & 23 & 0.029 \\
\hline & Negative & $33(49 \%)$ & 21 & 12 & \\
\hline \multirow[t]{2}{*}{ T-stage } & T1-T2 & $32(47 \%)$ & 19 & 13 & 0.15 \\
\hline & T3-T4 & $36(53 \%)$ & 14 & 22 & \\
\hline \multirow[t]{2}{*}{ Stage Group } & Early & $24(35 \%)$ & 13 & 11 & 0.66 \\
\hline & Advanced & $44(65 \%)$ & 20 & 24 & \\
\hline \multirow[t]{4}{*}{ Histological grade } & G1 & $17(25 \%)$ & 10 & 7 & 0.76 \\
\hline & G2 & $46(68 \%)$ & 21 & 25 & \\
\hline & G3 & $3(4 \%)$ & 1 & 2 & \\
\hline & Unknown & $2(3 \%)$ & 1 & 1 & \\
\hline
\end{tabular}

NLR: Neutrophils-to-Lymphocytes Ratio; PS: Performance Status; LN: Lymph Nodes; N/A: Not Available. 
used for estimation of survival rates. The level of significance was set at $p \leq 0.05$. The chi-square test was used to determine the associations between the pre-treatment NLR and different variables. The impact of NLR on overall (OS) and progression free survivals (PFS) was investigated in both uni-and multivariate analyses.

\section{Results}

Mean follow up time was 19.5 months (SD \pm 19.8 ). 3 -years OS and PFS were $61.6 \%$ and $39.7 \%$, respectively. Oral Tongue as a primary site represents about half of the patients 35 (51.4\%), followed by alveolar ridge $(14.7 \%)$ and floor of mouth $(13.2 \%)$, buccal mucosa (10.3\%) and other sites (10.3\%).

The median pretreatment NLR ratio was 1.79 . According to the median NLR as a cut-off value, patients were classified into two groups: high NLR and low NLR.

The correlations between NLR and selected variables showed that elevated NLR was significantly associated with male gender $(p=0.003)$ and with the presence lymph nodes (LN) metastases $(p=0.029)$ (Table 1$)$.

\section{Survival analysis}

In our patients 3-years OS and PFS were significantly better for patients with low NLR compared to high NLR (69.5\% vs. $54.7 \% p=0.037$ ) (Figure 1 ). Three-years PFS was $51 \%$ vs. $29.5 \%$ ( $p=0.028)$ for low and high NLR, respectively (Figure 2). Table 2 showed also the univari- ate analysis of the other prognostic factor. Multivariate analysis confirmed NLR is an independent prognostic factor for both OS $(p=0.005)$ and PFS $(p=0.008)$ (Table $3)$.

\section{Discussion}

Prognostic significance of NLR as a systemic inflammatory marker in various solid malignancies has been postulated. A recent systematic review and meta-analysis of studies with over 40,000 patients has demonstrated a negative correlation between high NLR and PFS as well as OS [14]. Furthermore, prognostic significance of NLR in the nasopharyngeal cancers has been highlighted in some recent publications from the Far East $[15,16]$. However, role of NLR in OSCC has largely remained unexplored.

The link between inflammation and cancer is widely accepted. Direct and indirect evidences for this link include: 1) The risk of developing some cancer increases with inflammatory diseases (e.g. bladder, cervical, gastric, esophageal cancers). 2) Non-steroidal anti-inflammatory drugs reduce the risk of developing certain cancers (e.g. colon cancer). 3) Signaling pathways involved in inflammation operate downstream of oncogenic mutations (e.g. mutations in the genes encoding RAS, MYC and RET). 4) Adoptive transfer of inflammatory cells or over expression of inflammatory cytokines promotes the development of tumors [17]. NLR can be

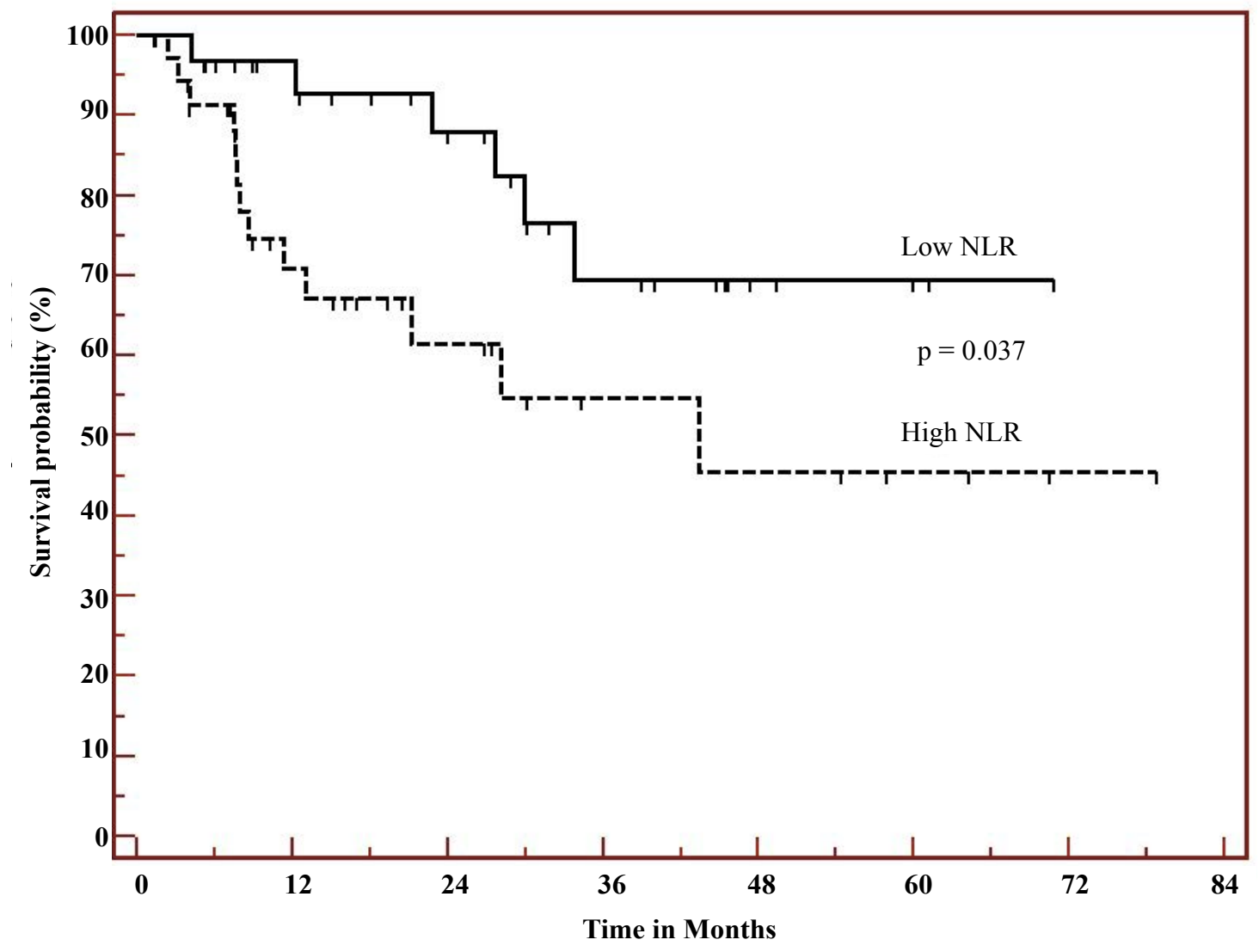

Figure 1: Overall survival according to Neutrophil-to-Lymphocyte Ratio, High NLR (> 1.79) and Low NLR (<1.79). 


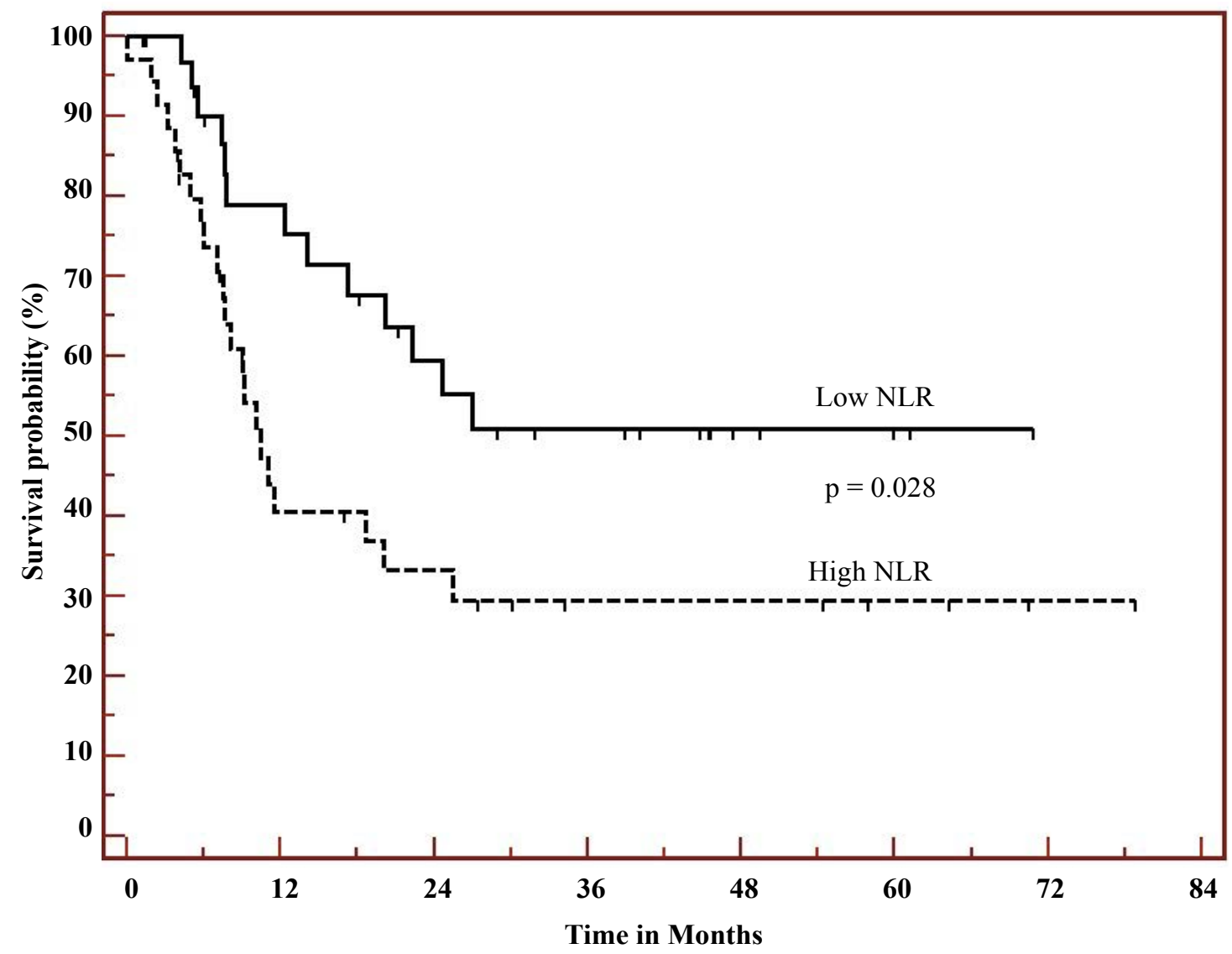

Figure 2: Progression free survival according to Neutrophil-to-Lymphocyte Ratio, High NLR (> 1.79) and Low NLR (<1.79).

Table 2: Univariate analysis for 3-years overall and progression free survival in relation to different prognostic factors.

\begin{tabular}{|c|c|c|c|}
\hline Variables & & 3-Y OS & 3-Y PFS \\
\hline \multirow[t]{3}{*}{ AGE } & $<65$ & $69.3 \%$ & $46.5 \%$ \\
\hline & $\geq 65$ & $48.3 \%$ & $28.3 \%$ \\
\hline & & $p=0.023$ & $p=0.05$ \\
\hline \multirow[t]{3}{*}{ SEX } & $M$ & $57.8 \%$ & $41.0 \%$ \\
\hline & $F$ & $67.0 \%$ & $38.0 \%$ \\
\hline & & $p=0.79$ & $p=0.88$ \\
\hline \multirow[t]{3}{*}{ PS } & $<2$ & $74.0 \%$ & $51.5 \%$ \\
\hline & $\geq 2$ & $40.7 \%$ & $20.5 \%$ \\
\hline & & $p=0.004$ & $p=0.006$ \\
\hline \multirow[t]{4}{*}{ Tobacco } & Yes & $70.3 \%$ & $44.0 \%$ \\
\hline & NO & $63.3 \%$ & $36.7 \%$ \\
\hline & N/A & $51.3 \%$ & $42.6 \%$ \\
\hline & & $p=0.56$ & $p=0.85$ \\
\hline \multirow[t]{3}{*}{ LN status } & Negative & $85.6 \%$ & $62.8 \%$ \\
\hline & Positive & $37.2 \%$ & $20.1 \%$ \\
\hline & & $p=0.001$ & $p=0.001$ \\
\hline \multirow[t]{3}{*}{ T-stage } & T1-T2 & $77.2 \%$ & $51.3 \%$ \\
\hline & T3-T4 & $43.0 \%$ & $27.6 \%$ \\
\hline & & $p=0.017$ & $p=0.056$ \\
\hline \multirow[t]{3}{*}{ Stage Group } & Early (I/II) & $88.1 \%$ & $61.4 \%$ \\
\hline & Advanced (III/IV) & $43.8 \%$ & $27.0 \%$ \\
\hline & & $p=0.009$ & $p=0.013$ \\
\hline \multirow[t]{5}{*}{ Histological grade } & G1 & $79.4 \%$ & $68.0 \%$ \\
\hline & G2 & $59.0 \%$ & $30.9 \%$ \\
\hline & G3 & $0.0 \%$ & $0.0 \%$ \\
\hline & Unknown & $50.0 \%$ & $50.0 \%$ \\
\hline & & $p=0.026$ & $p=0.07$ \\
\hline NLR median & High NLR & $54.7 \%$ & $29.5 \%$ \\
\hline
\end{tabular}

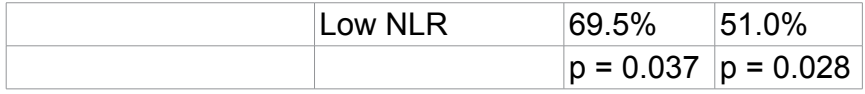

OS: Overall Survival; PFS: Progression Free Survival; NLR: Neutrophils-to-Lymphocytes Ratio; PS: Performance Status; LN: Lymph Nodes; N/A: Not Available.

considered to be a marker representing presence of inflammatory response.

The mechanism of association between NLR and prognosis of cancer is not fully understood but some of the available evidence does offer some understanding. For instance high neutrophil count was found to inhibit the cytolytic activity of immune cells such as lymphocytes, activated T cells, and natural killer cells and consequently suppresses the immune system $[18,19]$. Tumor associated neutrophils have been shown to cause stimulation of tumor microenvironment by the release of tumor growth promoting agents like matrix metalloproteinases [20] and fibroblast growth factor that result in migration of endothelial cells and dissociation of tumor cells. The ultimate results will be enhancement of angiogenesis, tumor growth, and development of metastases. Neutrophils also inhibit apoptosis of the tumor cells by activation of nuclear factor (NF)-KB [21].

In contrast, lymphocytes have an important role in cytotoxic cell death and cytokines production that inhibit proliferation and metastatic activity of tumor cells [10]. Increased tumor infiltrating lymphocyte has been correlated with a better prognosis. Tumor infiltration by 
Table 3: Cox-Regression multivariate analysis for overall and progression free survivals.

\begin{tabular}{|l|l|l|l|l|}
\hline & Overall Survival & & \multicolumn{2}{l|}{ Progression free Survival } \\
\hline Covariate & $\mathrm{P}$ & $95 \% \mathrm{Cl}$ of Exp (b) & $\mathrm{P}$ & $95 \% \mathrm{Cl}$ of Exp (b) \\
\hline Age & 0.0424 & 1.0515 to 12.1637 & 0.1372 & 0.8281 to 4.0439 \\
\hline PS & 0.0006 & 3.2832 to 71.4708 & 0.0019 & 1.6409 to 8.6963 \\
\hline Grading & 0.0013 & 1.4295 to 4.3069 & -- & --- \\
\hline T-stage & 0.1337 & 0.0448 to 1.5002 & 0.1193 & 0.1314 to 1.2547 \\
\hline N-stage & 0.2076 & 0.3835 to 86.5452 & 0.4386 & 0.3973 to 8.4978 \\
\hline Stage Group & 0.3875 & 0.1342 to 182.3538 & 0.1902 & 0.5133 to 29.8528 \\
\hline NLR & 0.0052 & 1.9445 to 41.7779 & 0.0085 & 1.3437 to 7.2873 \\
\hline
\end{tabular}

$\mathrm{P}=\mathrm{P}$ value; $\mathrm{Cl}$ : Confidence Interval; NLR: Neutrophils-to-Lymphocytes Ratio; PS: Performance Status.

lymphocytes has been reported to indicate the generation of an effective antitumor cellular immune response [22].

Our present study demonstrates a positive correlation between a low pretreatment NLR andsurvival outcomes for progression free survival in patients with non-metastatic OSCC. These findings are consistent with other reports in OSCC [11,12].

In the absence of universal agreement about the cutoff value to define low and high NLR we used the median NLR (1.79) as a cut-off value. The way of choosing cut-off values differs among studies. Cut-off values on the basis of the median [12], higher quartile [5] or values determined by the use of receiver operating characteristic (ROC) curve analysis [10] were used. As a result, different cutoff values are reported however, the vast majority of cut-offs were in the ranges of 2 to 4 . Nonetheless, the optimal cut-off levels for NLR remains to be established.

In our work high NLR was associated with both male sex and the presence of LN metastases. The association between high NLR with poor prognostic features like positive nodal involvement has been observed in breast cancer patients [23]. We can speculate that our findings are due to similar reasons as described in other tumor types. Although inmost studies, no difference in NLR between both genders was found, however, few studies are concordant with ours showing male sex predominance of high NLR [7,24].

There is a paucity of data exploring association of NLR in OSCC and our results can add a value in the support of the existing data. To our knowledge this is the first work exploring this issue in our population. This may be of particular value especially in view of the presence of the racial difference in the average NLR $[25,26]$.

The limitations of our study areits retrospective nature and the limited number of patients. Prospective studies with larger number cases are needed to further clarify this issue and to accurately define the cut-off value to discriminate the prognostic group.

\section{Conclusions}

Pretreatment NLR is an independent prognostic factor for patients with OSCC. It provides a simple, cheap and early predictor of outcome in this group of patients. Larger and prospective studies are required to determine the optimal cut-off values to accurately identify NLR related risk groups.

\section{References}

1. Fitzmaurice C, Allen C, Barber RM, Barregard L, Bhutta $Z A$, et al. (2017) Global, regional, and national cancer incidence, mortality, years of life lost, years lived with disability, and disability-adjusted life-years for 32 cancer groups, 1990 to 2015: a systematic analysis for the global burden of disease study. JAMA oncology 3: 524-548.

2. Taghavi N, Yazdi I (2015) Prognostic factors of survival rate in oral squamous cell carcinoma: Clinical, histologic, genetic and molecular concepts. Arch Iran Med 18: 314-319.

3. Salesiotis AN, Cullen KJ (2000) Molecular markers predictive of response and prognosis in the patient with advanced squamous cell carcinoma of the head and neck: evolution of a model beyond TNM staging. Curr Opin Oncol 12: 229239.

4. Feller L, Altini M, Lemmer J (2013) Inflammation in the context of oral cancer. Oral oncology 49: 887-892.

5. Azab B, Bhatt VR, Phookan J, Murukutla S, Kohn N, et al. (2012) Usefulness of the neutrophil-to-lymphocyte ratio in predicting short-and long-term mortality in breast cancer patients. Ann Surg Oncol 19: 217-224.

6. Yamanaka T, Matsumoto S, Teramukai S, Ishiwata $\mathrm{R}$, Nagai $Y$, et al. (2007) The baseline ratio of neutrophils to lymphocytes is associated with patient prognosis in advanced gastric cancer. Oncology 73: 215-220.

7. Chen S, Zhang L, Yan G, Cheng S, Fathy AH, et al. (2017) Neutrophil-to-lymphocyte ratio is a potential prognostic biomarker in patients with ovarian cancer: a meta-analysis. Biomed Res Int 2017: 7943467.

8. Zhang GM, Zhu Y, Gu WJ, Zhang HL, Shi GH, et al. (2016) Pretreatment neutrophil-to-lymphocyte ratio predicts prognosis in patients with metastatic renal cell carcinoma receiving targeted therapy. International journal of clinical oncology 21: 373-378.

9. Tomita M, Shimizu T, Ayabe T, Yonei A, Onitsuka T (2011) Preoperative neutrophil to lymphocyte ratio as a prognostic predictor after curative resection for non-small cell lung cancer. Anticancer Res 31: 2995-2998.

10. An X, Ding PR, Wang FH, Jiang WQ, Li YH (2011) Elevated neutrophil to lymphocyte ratio predicts poor prognosis in nasopharyngeal carcinoma. Tumor Biology 32: 317-324.

11. Perisanidis C, Kornek G, Pöschl PW, Holzinger D, Pirklbauer K, et al. (2013) High neutrophil-to-lymphocyte ratio is an independent marker of poor disease-specific survival in patients with oral cancer. Medical Oncology 30: 334. 
12. Nakashima $H$, Matsuoka $Y$, Yoshida R, Nagata M, Hirosue A, et al. (2016) Pre-treatment neutrophil to lymphocyte ratio predicts the chemoradiotherapy outcome and survival in patients with oral squamous cell carcinoma: a retrospective study. BMC cancer 16: 41.

13. Chen S, Guo J, Feng C, Ke Z, Chen L, et al. (2016) The preoperative platelet-lymphocyte ratio versus neutrophillymphocyte ratio: which is better as a prognostic factor in oral squamous cell carcinoma?. Therapeutic advances in medical oncology 8: 160-167.

14. Templeton AJ, McNamara MG, Šeruga B, Vera-Badillo FE, Aneja P, et al. (2014) Prognostic role of neutrophil-to-lymphocyte ratio in solid tumors: a systematic review and meta-analysis. JNCl: J Natl Cancer Inst 106.

15. Takenaka Y, Kitamura T, Oya R, Ashida N, Shimizu K, et al. (2017) Prognostic role of neutrophil-lymphocyte ratio in nasopharyngeal carcinoma: A meta-analysis. PLoS One 12: e0181478.

16. Yin J, Qin Y, Luo YK, Feng M, Lang JY (2017) Prognostic value of neutrophil-to-lymphocyte ratio for nasopharyngeal carcinoma: A meta-analysis. Medicine 96: e7577.

17. Mantovani A, Allavena P, Sica A, Balkwill F (2008) Cancer-related inflammation. Nature 454: 436-444.

18. Shau HY, Kim A (1988) Suppression of lymphokine-activated killer induction by neutrophils. The Journal of Immunology 141: 4395-4402.

19. El-Hag A, Clark RA (1987) Immunosuppression by activated human neutrophils. Dependence on the myeloperoxidase system. The Journal of Immunology 139: 2406-2413.
20. Shamamian P, Schwartz JD, Pocock BJ, Monea S, Whiting D, et al. (2001) Activation of progelatinase A (MMP-2) by neutrophil elastase, cathepsin $\mathrm{G}$, and proteinase-3: A role for inflammatory cells in tumor invasion and angiogenesis. Journal of cellular physiology 189: 197-206.

21. Noh H, Eomm M, Han A (2013) Usefulness of pretreatment neutrophil to lymphocyte ratio in predicting disease-specific survival in breast cancer patients. J Breast Cancer 16: 5559.

22. Denkert C, Loibl S, Noske A, Roller M, Müller BM, et al. (2009) Tumor-associated lymphocytes as an independent predictor of response to neoadjuvant chemotherapy in breast cancer. Journal of clinical oncology 28: 105-113.

23. Faria SS, Fernandes Jr PC, Silva MJ, Lima VC, Fontes W, et al. (2016) The neutrophil-to-lymphocyte ratio: a narrative review. ecancermedicalscience 10: 702.

24. Grenader T, Waddell T, Peckitt C, Oates J, Starling N, et al. (2016) Prognostic value of neutrophil-to-lymphocyte ratio in advanced oesophago-gastric cancer: exploratory analysis of the REAL-2 trial. Ann Oncol 27: 687-692.

25. Azab B, Camacho-Rivera M, Taioli E (2014) Average values and racial differences of neutrophil lymphocyte ratio among a nationally representative sample of United States subjects. PLoS One 9: e112361.

26. Kweon OJ, Lee MK, Kim HJ, Chung JW, Choi SH, et al. (2016) Neutropenia and neutrophil-to-lymphocyte ratio in a healthy Korean population: race and sex should be considered. Int J Lab Hematol 38: 308-318. 